\title{
La brièveté dans la forme courte : D'Art d'art sur France 2
}

Jean-Bernard Cheymol

Lorsque les analystes des médias ou les présentateurs évoquent la brièveté des programmes, c'est souvent pour regretter que l'on ne puisse pas parler plus longuement du sujet de l'émission. Victime de préjugés, la brièveté est en effet souvent perçue sous son seul versant négatif, comme un abrègement par rapport à un discours long, dont on déplorerait qu'il soit malheureusement impossible dans le contexte médiatique actuel, en référence à une exhaustivité qu'elle viendrait entamer ou rompre, origine perdue qui a tout du mythe. C'est a fortiori le cas à la télévision, où il y a non seulement juxtaposition des messages brefs comme dans un recueil de textes ou d'images, mais succession rapide de messages qui s'effacent rapidement, selon la logique de la dissipation analysée par Jean-Pierre Esquenazi ${ }^{1}$, du moins dans les moments de télévision dans lesquels s'inscrit la diffusion de D'Art d'art, au milieu des plages de publicité, du bulletin météorologique, d'annones de programmes et d'autres émissions courtes. Par exemple, pour Pierre Bourdieu, l'urgence et le manque de temps obligent les fast-thinkers à proposer du fast-food culturel, de la «nourriture culturelle prédigérée, pré-pensée $»^{2}$.

On peut penser qu'un tel a priori péjoratif occulte le phénomène constitué par le discours télévisuel bref, dès lors que l'on voit dans la brièveté la caractéristique strictement dimensionnelle d'un format court, trop court eu égard au contenu qui le dépasse. Or, au-delà du constat de leur dimension réduite par rapport à celui d'autres formes télévisuelles, la brièveté n'est pas seulement l'opposé d'un long préexistant, qu'elle viendrait limiter, abréger, elle a sans doute une signification intrinsèque, elle peut faire sens par elle-même au lieu

\footnotetext{
${ }^{1}$ Esquenazi, Jean-Pierre, TF1, Le pouvoir d'un média, Paris, L'Harmattan,

${ }^{2}$ Bourdieu, Pierre, Sur la télévision, p. 31.
} 
d'être seulement une limitation dans la transmission d'un sens préexistant dans un contenu. Chercher ce sens suppose d'élargir la compréhension de la brièveté, en la dégageant de son sens quantitatif ou dimensionnel. D'où l'intérêt, d'étudier plus précisément un cas de discours médiatique bref, celui sur les arts plastiques, dans une émission courte, D'Art d'art, sur France 2, une émission d'une minute trente consacrée à une œuvre d'art plastique) où est favorisée la découverte, souvent fortuite, au hasard du zapping, d'une œuvre d'art au sujet de laquelle Frédéric Taddeï, le présentateur, fait le récit d'une anecdote. L'objectif de cet article est de montrer comment différentes conceptions de la brièveté sont impliquées ou à l'œuvre dans les émissions courtes. Seront distinguées deux manières d'appréhender la brièveté : l'approche dimensionnelle du bref comme court, que l'on peut qualifier de classique, et une approche non dimensionnelle de celui-ci - qui n'invalide pas la première - dans les émissions courtes.

\section{Une approche dimensionnelle et spatio-temporelle du bref comme}

\section{court}

Prise sous l'angle quantitatif et dimensionnel, spatio-temporel, en un sens donc non bergsonien, la durée est une quantité de temps spatialisé, converti en étendue mesurable. Le temps est alors conçu en termes quantitatifs, la brièveté représentant une quantité réduite de temps, une durée courte, une spécificité de la brièveté télévisuelle étant que la petite quantité s'y trouve associée à un effacement, à une évanescence constitutive du bref dans un média de flux.

Plusieurs stratégies d'adaptation à cette contrainte du format court télévisuel, dont le contenu est foncièrement menacé d'inexistence, sont à l'œuvre. Il est possible de restreindre délibérément le contenu du message, en limitant la recherche d'une signification profonde de l'œuvre, pour s'attarder sur des détails souvent anecdotiques. Mais on peut aussi donner le sentiment de le densifier ou de le condenser ; on chercherait alors à faire retenir le message, tentant ainsi d'arrêter le cours du temps, de lutter contre la perte qui risque 
d'accompagner la brièveté, grâce à divers procédés qui visent à densifier le présent ou, du moins, à donner une illusion de plus grande densité du discours. L'émission brève pourrait dès lors être perçue par le téléspectateur comme une source de gain de temps, particulièrement précieuse pour l'individu contemporain obsédé par les risques de perte.

\section{De la retenue...}

La première stratégie discursive à envisager consiste, de la part du média télévisuel, à s'accommoder de la brièveté en restreignant l'ambition explicative du sens de l'œuvre, en une forme de retenue, qui, expurgée de son contenu moral, désigne le choix de rester dans l'inaccomplissement.

Ainsi, dans le discours verbal du présentateur, l'allusion fréquente à la difficulté de comprendre l'œuvre d'art participe d'un avortement des velléités de compréhension de son sens, à l'œuvre dans D'Art d'art. Dans l'émission, foncièrement inchoative, un tel court-circuitage de la reconnaissance intellectuelle s'accompagne, par instants, d'une accentuation de la sensation, grâce à l'usage du ralenti : de façon furtive mais insistante, les émissions donnent l'image ou le signe d'une perception sensorielle de l'œuvre, sans rapport avec le commentaire, sans cesse débutante et recommençante, puisque l'on assiste à une série de parcours visuels, brefs mais au ralenti, au sein de l'œuvre, depuis des points de départ très différents. L'émission courte apparait, en ce sens, comme un moment où le flux télévisuel, de même que l'écoulement temporel en général, peut, par un redoublement paradoxal de la brièveté, être ralenti, en une accentuation de l'attention aux commencements et, par là, au présent.

\section{... à la rétention}

Il existe aussi des stratégies qui consistent plutôt qu'à se retenir d'entrer dans un processus long, de compréhension d'un sens profond, en l'occurrence, à faire retenir le message, pour lutter contre sa dissipation. On passe alors de la retenue à la rétention, via des stratégies d'inscription dans la durée par 
densification ou condensation du sens. On peut ainsi densifier le message transmis par l'émission pédagogique sur l'art, pour en faire retenir ou mémoriser le maximum par le téléspectateur ou pour donner l'impression qu'il y a beaucoup dans ce qui est dit ou montré, en chargeant le contenu de l'émission d'une densité particulière. Durer consiste alors à subsister, comme lesté du poids lié à la densité d'un contenu sémantique.

Plusieurs techniques sont ici à distinguer. Prendre pour sujet une œuvre d'art constitue déjà en soi une manière de s'extraire du temps court. Puisque l'œuvre est un condensé de sens, que l'observation ne peut jamais parvenir à sonder dans son exhaustivité. Déjà, Sénèque écrivait de l'œuvre d'art qu'elle fonctionnait sur le principe de la condensation : «C'est le propre d'un grand artiste de savoir enfermer le tout dans un espace insignifiant ${ }^{3}$. L'art en général travaillerait sur le modèle de la miniaturisation, qui renverse le rapport usuel entre le petit et le grand. Il est également possible d'accroître la quantité de sens présent dans un temps limité grâce à des stratégies de condensation temporelle, dans le récit, comme dans l'émission consacrée à La Vierge de Donatello qui souligne la présence d'un instant prégnant, tel que défini par Lessing, au sein de l'œuvre :

Regardez comme ils (la vierge et l'enfant) débordent de présence, et ce n'est pas tout : on peut pénétrer dans les pensées de la vierge. Son visage n'est pas neutre, il n'est pas béat, il est terrible au contraire comme si en contemplant son enfant, sa mère voyait déjà tout ce qui va lui arriver. C'est la passion du Christ tout entière qui défile devant les yeux de Marie. De quoi prier pendant des siècles.

Le tableau représente un instant que l'on peut qualifier de prégnant, au sens où il condense le temps à venir. Il l'est même en un sens renforcé du terme, puisqu'il condense non seulement un futur proche, mais aussi, d'après le présentateur, un avenir particulièrement lointain. C'est en effet la vie du Christ,

\footnotetext{
${ }^{3}$ Sénèque, Lettres à Lucilius, 53, 11.
} 
mais, au-delà, implicitement, de l'humanité tout entière, qui le prie, qui est à lire d'après Frédéric Taddeï dans La Vierge de Donatello. Dans ce jeu sur le temps, on assiste à l'inscription dans une forme brève du temps le plus long qui soit, celui de la scène sans cesse rejouée, encore aujourd'hui, par les chrétiens, la vie du Christ. Un point cristallise tous les espoirs et toutes les craintes, en lui se projette l'avenir. En si peu de temps, en un simple regard la destinée de l'humanité tout entière, y compris métaphysique, semble se jouer. La condensation emploie bien d'autres voies mais, dans l'ensemble, elle contribue à une densification des instants brefs au sein des émissions courtes sur l'art, susceptibles de les inscrire dans le temps long. Joue alors dans D'Art d'art une économie de la signification, en réponse à une contrainte, la durée courte, qui correspond à l'économie la logique classique de la concision, mais dans le contexte du flux télévisuel. Toutefois, à la limite de la condensation, mais en vertu de sa logique propre, se tient le risque de l'excès.

\section{La condensation jusqu'à l'excès}

En effet, le court, dès lors qu'il est, par sa définition même, affecté d'un manque, est toujours foncièrement trop court, ce qui se traduit dans l'émission par une intensification de la condensation jusqu'à l'excès, où le contenu en vient à déborder la forme dans laquelle il est enclos. La condensation peut renvoyer à un trop plein, au débordement d'un sens qui peine à s'exprimer dans la forme brève et qui peut se traduire par deux phénomènes différents, voire opposés, qui en sont les deux facettes : une accumulation de visions fugitives et partielles et, d'autre part, un manque affiché, dans l'évocation en creux, notamment grâce au procédé de l'ellipse, de l'infini du sens, une insuffisance manifeste du discours, en comparaison de celui-ci. L'émission brève joue alors sur la quantité, à la fois sur le trop ou le pas assez, qui y alternent. 
L'accumulation de visions partielles et fugitives de l'œuvre d'art, selon des points de vue différents, produit un sentiment d'excès, par exemple lorsqu'à plusieurs reprises dans D'Art d'art on s'en approche depuis des points qui varient d'un moment à l'autre. Dans l'émission consacrée à La coupe de fruits de Lubin Baugin, on peut ainsi compter jusqu'à treize apparitions différentes de l'œuvre étudiée. Sans doute s'agit-il de tenter de combler le regard par une accumulation de vues fragmentaires du même objet où, faute de parvenir à le donner entièrement à voir, on en multiplie les approches. Par une rapidité accrue des mouvements de caméra, qui donne un sentiment de saturation et d'excès, on va jusqu'à rendre l'œuvre comme in-visible par une surcharge d'apparitions à l'écran. Faute de donner à observer, on donne une accumulation de visions qui saturent le regard tout en rendant l'objet inobservable.

Un autre procédé où est sensible une forme d'excès, censé dépasser peutêtre les limites de l'émission, est la figure de l'ellipse, qui peut être considérée comme un pendant de l'accumulation, dans la mesure où, en indiquant l'idée d'une insuffisance ou d'un manque, renvoie négativement à l'infini, ici du sens de l'œuvre d'art. Lorsque l'on montre notamment une œuvre qui a un lien avec la religion, par exemple la Mère supérieure de Tamara de Lempicka, souvent, cette dernière est animée d'un mouvement de rotation, qui non seulement rend l'œuvre inobservable, mais suscite une forme de vertige chez le téléspectateur. Il est impossible à un visiteur de musée de se déplacer aussi rapidement, les mouvements en tous sens de la caméra ne peuvent correspondre à ceux d'un observateur humain Est suggéré un au-delà du regard, en une forme de « regard surréel $»^{4}$, selon l'expression de Guy Lochard et Jean-Claude Soulages. De plus, ce mouvement, qui a un caractère désordonné, intervient au moment où Frédéric Taddeï s'interroge sur les raisons, bien mystérieuses - il ne donne pas de

${ }^{4}$ Lochard, Guy, Soulages, Jean-Claude, «Faire voir la parole», in La télévision, Les débats culturels, «Apostrophes ». 
réponse à sa question -, qui ont bien pu pousser Tamara de Lempicka à quitter son domaine de prédilection, la belle société des Années Folles, pour peindre une religieuse. Il évoque alors «l'angoisse de la page blanche » pour expliquer le passage de Tamara de Lempicka à ce sujet religieux. Or - ce qui constitue un nouveau procédé - au moment où il prononce ces mots, apparaît à l'écran l'image du mur sur lequel est accroché la toile et sur lequel celle-ci semble bien petite, un simple point dans un univers qui la dépasse, comme si ce mur était luimême une image de la page blanche, de l'indéterminé. Sans doute est-ce là encore une façon de suggérer l'infini, celui des possibles, des explications de la décision, devant lequel on ne peut qu'avoir le vertige. D'Art d'art met ainsi en scène un mystère de la création artistique. Cela n'est sans doute pas un hasard si l'infini dans la création et son mystère est suggéré à l'occasion de la présentation d'un tableau au sujet religieux.

D'autres traits de l'émission D'Art d'art renvoient à l'infini. Le présentateur, qui se trouve sur un des plateaux reliés par les escaliers, semble comme inséré dans un espace virtuel potentiellement infini. Le zoom avant, assez fréquemment employé dans les deux émissions, donne parfois le sentiment au téléspectateur d'être plongé dans un espace infini et que le rapprochement, ou la plongée dans le détail peuvent se poursuivre indéfiniment, s'ils n'étaient arrêtés à un moment donné. Dans ce cas, l'approche éloigne ou fait «le jeu du lointain », selon Maurice Blanchot, car elle donne l'impression qu'il y a toujours davantage à voir. Dans D'Art d'art consacrées au Monochrome IKB 3 d'Yves Klein, le détail ouvre sur l'infiniment petit du grain, mais le bleu se révèle être aussi celui d'un océan. En particulier, l'alternance entre les plans où l'œuvre est tantôt éloignée, tantôt au contraire agrandie, entre zoom arrière et zoom avant, dans certaines émissions, crée ce sentiment de réversibilité de l'infime et de l'immense, constitutif du jeu sur l'excès de sens dans la brièveté. Certaines disproportions sont également significatives, comme celle entre le présentateur et l'œuvre, en un renversement étonnant des rapports naturels de grandeur. 
Grâce à tous ces procédés, est procuré le sentiment d'un débordement, l'impression qu'il y a beaucoup plus, si ce n'est dans l'émission elle-même, du moins dans l'œuvre, et le suggérer suffit à donner de la densité à ce qui est montré, donc à l'émission elle-même. La présence récurrente de l'ellipse dans les émissions est une manière de signifier que le sens d'une œuvre d'art, en l'occurrence, excède la durée de l'émission, ce qui constitue sans doute une manière pour nos émissions de contourner la contrainte de durée courte. Toutefois, la désignation d'un excès de sens, en même temps que le signe salutaire d'un au-delà des limites imposées par le format de l'émission, sonne aussi inévitablement comme un aveu d'impuissance : le discours télévisuel court serait trop court, eu égard à son contenu. Peut être la condensation n'est-elle alors qu'un pis aller, un palliatif faible, voué à s'abîmer dans l'impuissance de l'excès, signifiant en cela une incapacité structurelle du média à dire le sens, dont il jouerait en la désignant.

\section{II. une brièveté non dimensionnelle : le bref distinct du court}

Or les émissions brèves sur l'art ne sont pas brèves en ce seul sens négatif, qui justifie les tentatives d'adaptation à une contrainte du court que l'on vient d'évoquer. Dans ces formes brèves médiatiques, identifiées grâce à leur durée courte, la brièveté ne se réduit pas à sa dimension quantitative évidente. Le bref n'est pas le court, même si les deux adjectifs ont été associés dans l'histoire de la langue 5 . Outre ce qui dure peu, il désigne l'éphémère qui, dans sa discontinuité, ne dure pas, c'est-à-dire ne subsiste pas ou ne se conserve pas tel quel, selon un sens dimensionnel, non bergsonien, de la durée comme quantité de temps mesurable et conservation de soi à l'identique. La brièveté renvoie au fugitif, au fugace : le bref peut être le signe de ce qui se dérobe, de ce sur quoi

\footnotetext{
${ }^{5}$ Il revient à Gérard Dessons d'avoir mis en évidence la différence entre le cour et le bref dans la littérature et dans l'analyse littéraire, dans un article intitulé « la notion de brièveté ».
} 
l'on passe, dans lequel on entre à moitié. « D'Art d'art » fait référence à l'expression « dare dare » qui, entre autres, suggère la nécessité de faire vite, à la sauvette, comme s'il fallait fuir. Le bref peut caractériser le passage d'un moment à l'autre. La brièveté se manifeste aussi dans la rupture, dans la discontinuité. Lorsque l'on demande d'abréger un discours, la brièveté impose une limite de fin, puisque l'abrègement porte sur la fin du discours, non sur son début. Le bref caractérise une inflexion temporelle, où une trajectoire se modifie, un processus s'interrompt. De même, chez Bachelard6, l'instant se caractérise par une discontinuité fondamentale, crée une rupture, refuse la continuité de la durée conçue comme subsistance..

Si l'on peut distinguer ces différentes formes de brièveté, il faut aussi préciser qu'elles ne se contredisent nullement mais, bien au contraire, sont complémentaires. Il existe un lien étroit entre elles. Tout d'abord, la durée courte s'inscrit en rupture d'un long préexistant, qu'il s'agisse du journal télévisé qui précède, des fictions qui suivent la diffusion de l'émission à ses débuts. Le court, dès lors qu'il rompt avec le long, tend à devenir bref. On rejoint ici la réflexion bergsonienne, selon laquelle la mesure du temps et donc La perception du court sont au fond qualitative. «Court» et «long» sont des prédicats qui, bien que portant sur la quantité, sont curieusement non quantitatifs mais qualitatifs en ce qu'ils n'ont de sens que relatif. Bergson montre qu'au fond de la quantité il y a du qualitatif: lorsque nous comptons des unités en les alignant dans l'espace, l'organisation de ces unités est un "processus tout dynamique, assez analogue à la représentation purement qualitative qu'une enclume sensible aurait du nombre croissant des coups de marteau »7. En mesurant le temps et en le caractérisant comme court ou comme long, en y introduisant la quantité, on oublie que le nombre, la multiplicité quantitative où se juxtaposent des unités extérieures les unes aux autres, s'enracinent dans une

Dans L'intuition de l'instant.

Bergson, Henri, Euvres, p. 82 (Essai sur les données immédiates de la conscience, p. 92). 
multiplicité de nature qualitative : «c'est donc grâce à la qualité de la quantité que nous formons l'idée d'une quantité sans qualité. »8 La conception dimensionnelle, de la brièveté tient ainsi à une tendance à concevoir que chaque instant vient s'inscrire dans le temps comme dans un cadre homogène préexistant, en raison de l'habitude qui consiste à développer le temps dans l'espace et à recouvrir la qualité par la quantité.

Mais le lien entre le court et le bref ne se réduit pas à cette brièveté du court. La forme courte peut aussi rendre plus sensible la brièveté comme rupture d'une continuité qui s'y manifeste. Au sujet du court métrage Un chien andalou, de Luis Buñuel, Thierry Méranger, dans, écrit : «Ce que la réussite d'Un chien andalou doit à la forme courte n'a pas été assez souligné. » 9 Car, entre autres, «la brièveté de l'œuvre rend perceptible la récurrence de certains motifs rayures, boîtes, poils, créatures androgynes - et accentue spectaculairement les bouleversements spatio-temporels. » Les ruptures dans la continuité sont plus perceptibles dans une forme courte que dans une longue; le court renforce la conscience de la fin, de la discontinuité du bref. Pour toutes ces raisons, les émissions étudiées sont donc indissociablement courtes et brèves.

\section{L'efficacité de la brièveté}

Dans ce contexte du court bref, le discours télévisuel bref sur les arts plastiques n'emprunte pas seulement la voie d'une rhétorique de la brièveté, de l'adaptation à une contrainte. En effet, l'efficacité du discours télévisuel bref sur les arts plastiques n'existe pas nécessairement malgré la brièveté, laquelle n'est pas seulement une une limite extérieure qui requiert des stratégies d'adaptation. Une conception dimensionnelle et négative du bref télévisuel, court à défaut d'être long, interdit de penser en quoi la brièveté télévisuelle fait sens, en ellemême, est créatrice de sens au lieu d'en faire passer un, tant bien que mal, par les fourches caudines du format réduit.

Ibid.

Méranger, Thierry, Le court métrage, p. 20. 
Il existe une efficacité propre de la brièveté pour offrir une relation nouvelle avec l'œuvre d'art. Se trouvant définie, non plus seulement comme durée courte, mais comme rupture dans une continuité, la brièveté est créatrice de possibilités, en l'occurrence de relation, de contact, elle modifie la relation traditionnelle entretenue avec l'œuvre d'art et de créer de nouvelles possibilités de contact avec elle. Cette capacité créatrice trouve sa source dans la nouveauté active de l'instantané dans la puissance de l'instant discontinu. Comme l'écrit Bachelard, pour qui la puissance de l'être s'exprime dans la discontinuité : «Cette puissance (...), c'est le retour à la liberté du possible, à ces résonances multiples nées de la solitude de l'être »10. De ce point de vue, le bref n'est pas du côté de la réalité qui viendrait contredire une possibilité, interrompre un processus, s'opposer à la réalisation du possible, mais il est intrinsèquement source d'ouverture, de création de possibilité. En l'occurrence, dans D'Art d'art, la brièveté a en elle-même pour effet de rendre plus facile la relation avec l'œuvre d'art, et ce de plusieurs façons.

On peut actualiser la relation dans la brièveté, notamment en la dramatisant, en jouant donc sur sa fin, grâce à l'évocation fréquente dans les récits du présentateur de la mort, de l'artiste, du destinataire de l'œuvre, des personnages représentés, de la maladie qui peut les affecter, des risques auxquels ils se trouvent confrontés, etc. Il s'agit alors de rendre la relation avec l'œuvre plus intense. On peut aussi, grâce à la brièveté, proposer une relation aléatoire, foncièrement occasionnelle avec l'œuvre d'art, faite de distance, de saisie fugitive d'occasions d'entrer seulement en contact avec elle. Notamment par le trait d'esprit dans les pointes du discours, qui, foncièrement bref, est selon Michel de Certeau un mode privilégié de la saisie de l'occasion. Les traits d'esprit sont fréquents dans le discours du présentateur de D'Art d'art et sont parfois surprenants, comme celui-ci :

« ce tableau de Millet, vous l'avez vu des milliers de fois »

$10 \quad$ Ibid., p.67. 
La brièveté peut même libérer le rapport à l'œuvre d'art, en introduisant l'empirique dans le discours et dans la relation avec l'œuvre. Cette dernière ne se constitue alors pas autour d'un centre d'attention légitime et peut prendre corps à partir de détails, dont le soulignement a souvent un effet désacralisant, le sens naissant de l'infrasémantique, en une expérience toujours différente d'une œuvre à l'autre. Dès lors qu'elle est occasionnelle, la relation avec l'œuvre d'art est plus facile, non seulement parce que l'émission courte solliciterait moins la mémoire, serait moins prenante pour l'esprit que la longue, mais parce que, dans la brièveté, la relation peut, libérée de tout présupposé, notamment de la nécessité de posséder des connaissances artistiques préalables, s'établir sur des bases toujours neuves. Ainsi, dans l'émission, la relation avec l'œuvre est rendue contingente, occasionnelle (dans tous les sens du terme), prenant place dans le cadre d'un opportunisme dont la brièveté est une condition essentielle. En effet, elle rend possible nouveau rapport au temps, où l'on cherche moins à inscrire sa relation à l'art dans la durée qu'à saisir les occasions11, les fameux kairoi de la pensée grecque, d'un contact éphémère avec telle ou telle œuvre, ce qui se manifeste entre autres par le recours fréquent au trait d'esprit. Dans cette disponibilité permanente de l'œuvre et de son spectateur, l'attente tend à disparaître, si bien que la brièveté crée une possibilité de relation fluctuante, au hasard des rencontres, en l'occurrence au hasard de celle qui a lieu dans ces moments de flux télévisuel. Dans cette forme de communication télévisuelle, la brièveté du discours redéfinit les termes, déplace l'enjeu de la relation de communication et y introduit de la flexibilité, donnant la possibilité à l'œuvre de s'ouvrir à un contact multiforme, plus simple à établir parce que limité par la brièveté, qui l'empêche de s'approfondir en un désir.

11 Les kairoi selon la conception de Valdimir Jankélévich, ou les instants propices dans la pensée chinoise du temps, auxquelles on peut faire référence dans la caractérisation de la brièveté télévisuelle de flux. 
En une forme de réduction, l'enjeu de la relation avec l'œuvre d'art peut alors se transformer, dans la brièveté : il peut ne plus s'agir de diffuser un savoir à son sujet, mais d'assurer de sa simple disponibilité pour un éventuel désir de la revoir, de la certitude de pouvoir entrer éventuellement un jour en contact avec une multitude d'objets culturels, certitude que recherche l'homme de l'âge de l'accès selon Jérémy Rifkin ${ }^{12}$. C'est le sens que prend aussi, selon nous, la dramatisation de la présence de l'œuvre, fréquente dans D'Art d'art, que je viens d'évoquer et où l'œuvre elle-même est souvent menacée de destruction, présentée comme ayant échappé à un tel risque, ou comme ayant résisté au temps, etc. L'œuvre peut être volée, comme La Joconde : le présentateur fait le récit du vol de l'œuvre en 1911 et de son retour au Musée du Louvre, en 1914. Entre-temps, elle était cachée dans une simple boîte, sous le lit du voleur. Dans cette relation brève, l'œuvre tend à n'exister que dès lors qu'elle est, potentiellement, en contact avec un public parce qu'elle menace de ne plus l'être.

Enfin, dans D'Art d'art, la relation peut se révéler d'autant plus facile qu'une distance plus grande avec l'objet d'art, peut-être définitive, peut aussi se créer. En effet, la dramatisation, lorsqu'elle porte sur l'existence de l'homme, quand l'artiste les personnages du tableau sont menacés de mort, en jouant sur la peur, loin de rapprocher, tient aussi paradoxalement à distance l'objet montré. Dans ce cas, le récepteur du discours télévisuel tend à s'identifier à un nonpublic, l'accès proposé est d'autant plus facile qu'il est voué à rester distant, la relation d'autant plus aisée à établir qu'elle est limitée au stade de l'accès même.

12 Rifkin, Jeremy, L'âge de l'accès. La nouvelle culture du capitalisme, Paris, La Découverte, 2005. 


\section{Conclusion}

Le média télévisuel, comme l'homme contemporain peut-être, semble partagé entre le désir de durer, de retenir l'écoulement temporel du flux télévisuel, et celui de surfer sur l'éphémère : cette tension est perceptible dans D'Art d'art. Car les deux attitudes, qui consistent pour l'une à vouloir retenir l'écoulement temporel que la brièveté rend plus sensible, pour l'autre à tirer parti de la brièveté, entendue en un sens non dimensionnel, coexistent dans les émissions brèves sur l'art, cette tension semblant constitutive du rapport au temps du média télévisuel. On peut dés lors se demander pourquoi coexistent des possibilités multiples de relation avec l'œuvre d'art qui reposent sur des rapports différents au temps, de rétention ou de jeu avec l'éphémère de l'occasion.

Il semble que le problème qui se pose aux émissions brèves soit de durer, malgré la brièveté et l'effacement incessant des messages. Et il est probable que tout soit bon pour cela, que tout converge pour réaliser le désir d'ancrage du discours médiatique. Toutefois, ce n'est pas seulement en vertu d'une coexistence extérieure mais également parce que la rupture, le changement sont aussi des manières de durer. En effet, selon Bachelard, mais aussi selon Bergson, qui se rejoignent sur ce point, pour durer, plutôt que de chercher à ancrer sa subsistance dans la densification de la substance, verbale ou iconique, accentuant le repli sur soi de l'être dans la substance, il est aussi nécessaire de créer, de rompre avec la continuité au lieu de vouloir s'y enraciner. La durée, pour Bachelard, se fonde sur une différence à soi dans l'acte bref plutôt que sur une identité close sur elle-même13. Dès lors, on ne dure pas malgré la brièveté, au contraire, on peut durer grâce à l'activité propre de cette dernière, source de création de possibilités nouvelles. C'est ce qu'exprime ainsi Vladimir Jankélévitch :

\footnotetext{
13 Il en va d'ailleurs de même pour Bergson, qui écrit : «Plus nous approfondirons la nature du temps, plus nous comprendrons que durée signifie invention, création de formes, élaboration continue de l'absolument nouveau. » (Bergson, Henri, L'évolution créatrice, p. 11).
} 
(...) la continuation même du devenir n'est possible que par la discontinuation du «survenir». La durée se constitue ainsi dans le sillage des événements soudains qui font devenir l'advenir et advenir le devenir et à tout instant fuser la nouveauté... ${ }^{14}$

La distinction que l'on a pu établir dans ce travail a ainsi une utilité classificatoire, mais repose sur une identité dialectique plus profonde.

\section{Bibliographie}

Bachelard, Gaston, L'intuition de l'instant, Paris, Stock, 1932.

Baier, Lothar, Pas le temps !, Traité sur l'accélération, Arles, Actes Sud, 2002.

Bergson, Henri, Euvres, Paris, PUF (Edition du centenaire), 1963.

Deleuze, Gilles, Le bergsonisme, Paris, PUF (« Quadrige »), 1966.

Dessons, Gérard, «La notion de brièveté », Poitiers, La Licorne, 21, 1991, p. 3-12.

Dessons, Gérard, «La stratégie du détail dans la critique d'art et la critique littéraire», in Pouvoir de l'infime, Saint-Denis, Presses universitaires de Vincennes, novembre 1997.

Dessons, Gérard, «La manière brève», in : La Forme brève, Actes du colloque francopolonais de Lyon, 19-21 décembre 1994, Paris, Champion, 1996.

Dessons, Gérard, «Brièveté et spécificité», Actes du colloque «Brièveté et narration brève», Université de Valéncia, Espagne, 28-30 avril 1998, Publicacions de l'abadia de Montserrat, 1998.

Domenget, Jean-Claude, «La multiplicité des temps télévisuels : de la production à la réception », in Vitalis André (dir.), «Les temps médiatiques», Temporalistes, $\mathrm{n}^{\circ}$ 42, décembre 2000.

Domenget, Jean-Claude, «Proposition d'étude des temporalités médiatiques », in Aroldi, Piermarco et Nicoletta, Vittadini (dir.), Attraversamenti, Communicazioni sociali, Anno XXV, ${ }^{\circ}{ }^{\circ}$, Milano, Universita Cattolica del Sacro Cuero, 2003, pp. 101-108.

Esquenazi, Jean-Pierre, Le pouvoir d'un media, TF1 et son discours, Paris, L'Harmattan, 1996. (principalement le chapitre « Flux et programmation »).

\footnotetext{
${ }^{14}$ Jankélévitch, Vladimir, Le Je-ne-sais-quoi et le Presque-rien, tome I, La manière et l'occasion, p. 116.
} 
Esquenazi, Jean-Pierre, «L'esthétique du plein », in Télévision : questions de formes (dir. Gardies, R. et Taranger, M.-C.), Actes du colloque d'Aix en Provence, 20-21 mai 1999, Paris, L'Harmattan, 2001, tome I, p. 85-94.

Lipovetsky, Gilles, L'empire de l'éphémère. La mode et son destin dans les sociétés modernes, Paris, Gallimard, 1987.

Lipovetsky, Gilles, Charles, Sébastien, Les temps hypermodernes, Paris, Grasset, 2004.

Roukhomovsky, Bernard, Lire les formes brèves, Paris, Nathan, 2001.

Rosenbourg, Harold, La tradition du nouveau, Paris, Editions de Minuit, 1962.

Rifkin, Jeremy, L’âge de l'accès. La nouvelle culture du capitalisme, Paris, La Découverte, 2005.

Willener, Alfred, A la lumière de la vitesse, Essai sur l'accélération du quotidien, Lausanne, Payot, 1990. 